New Concepts in Imaging: Optical and Statistical Models

D. Mary, C. Theys and C. Aime (eds)

EAS Publications Series, 59 (2013) 141-154

\title{
HIGH ANGULAR RESOLUTION AND YOUNG STELLAR OBJECTS: IMAGING THE SURROUNDINGS OF MWC 158 BY OPTICAL INTERFEROMETRY
}

\author{
J. Kluska ${ }^{1}$, F. Malbet ${ }^{1}$, J.-P. Berger ${ }^{2}$, M. Benisty ${ }^{1}$, B. Lazareff $^{1}$, \\ J.-B. Le Bouquin ${ }^{1}$ and C. Pinte ${ }^{1}$
}

\begin{abstract}
In the course of our VLTI young stellar object PIONIER imaging program, we have identified a strong visibility chromatic dependency that appeared in certain sources. This effect, rising value of visibilities with decreasing wavelengths over one base, is also present in previous published and archival AMBER data. For Herbig AeBe stars, the $\mathrm{H}$ band is generally located at the transition between the star and the disk predominance in flux for Herbig AeBe stars. We believe that this phenomenon is responsible for the visibility rise effect. We present a method to correct the visibilities from this effect in order to allow "gray" image reconstruction software, like Mira, to be used. In parallel we probe the interest of carrying an image reconstruction in each spectral channel and then combine them to obtain the final broadband one. As an illustration we apply these imaging methods to MWC158, a (possibly Herbig) B[e] star intensively observed with PIONIER. Finally, we compare our result with a parametric model fitted onto the data.
\end{abstract}

\section{Introduction}

The processes that lead to the formation of exoplanets are important to understand. Stars form after a collapse of a giant cloud of dust and gas. After a million year, a protoplanetary disk is forming around the star, believed to be the birthplace of planets.

\footnotetext{
${ }^{1}$ Institut de Planétologie et d'Astrophysique de Grenoble, UMR 5274, BP. 53, 38041 Grenoble Cedex 9, France

${ }^{2}$ ESO, Santiago Office, Alonso de Cordova 3107, Vitacura, Casilla 19001, Santiago de Chile, Chile
} 
A young star is surrounded by an active environment with which it interacts. Accretion disks (Monnier \& Millan-Gabet 2002), inner gaseous disks (Benisty et al. 2010; Eisner et al. 2009; Tannirkulam et al. 2008), infalling envelop renmands, winds (Cabrit et al. 2010; Dougados et al. 2005; Malbet et al. 2007; Tatulli et al. 2007) and jets (Cabrit 2003; Dougados et al. 2004) are the main components of such environments. There are several types of young stellar objects. The complexity of physical phenomena at play requires direct observation at the astronomical unit (A.U.) scale. Optical interferometry is able to bring such informations, because it can observe both in the near infrared, where the hot dust and hot gas nearby the star are emitting, and resolve the first A.U., which correspond to milliarcsecond scale at the distance of star formation regions.

Interferometry consists in combining the light of 2 or more telescopes in order to measure the complex degree of coherence. For that purpose, the interferometer measures interference fringes. The amplitude of the fringes yields the norm, and its position the phase of a complex quantity called visibility $V(u, v)$. Thanks to the van Cittert-Zernicke theorem we know that the Fourier transform of the visibilities in the Fourier plan $(u, v)$ gives us the intensity distribution $I(x, y)$ of the source. Unfortunately, in the near infrared (NIR) the atmosphere blurs the phases of the visibilities. The hint then, is to measure a quantity that is the sum of the phases over a baseline triangle. In that case, the atmospheric influence vanishes and we obtain only an astrophysical quantity called the closure phase. So, in practice, there are two interferometric measurements: the squared amplitude of the visibilities $V^{2}$ and the closure phases.

We noticed in several datasets that the visibility is higher at short wavelengths. If $B$ is a baseline length projected on the sky plan and $\lambda$ the wavelength, we can plot the squared visibilities $V^{2}$ in function of $B / \lambda$ (which is the spatial frequency). For a monochromatic object, we expect the points to follow a general trend in the visibility curve, since $B / \lambda$ represents only the spatial frequency. But, this is not the case. Indeed, we can see (Fig. 1) that the rising curve of visibilities per base is not fitting the general trend of the data for different baselines. First, it was seen in AMBER (Petrov et al. 2007) data, but it was considered as an instrumental defect. Now, the same effect has been observed with PIONIER (Le Bouquin et al. 2011). We try to explain this effect astrophysically, claiming that the image of the object is varying through the different spectral channels inside the same spectral band, and we propose three techniques in order to take it into account and to be able to reconstruct images.

These methods will be applied to an astrophysical object. They are useful to analyze MWC158 (also known as HD 50138). This star is a Be star known to have the $\mathrm{B}[\mathrm{e}]$ phenomenon and presents a strong variability (Andrillat \& Houziaux 1991; Borges Fernandes et al. 2009; Hutsemekers 1985; Pogodin 1997), which complexify the evolutionary stage identification of the source. Its distance is poorly constrained ( $d=500 \mathrm{pc} \pm 150 \mathrm{pc}$, van Leeuwen 2007).

In Section 2 we will describe the chromatic effects in the visibilities and the Section 3 will show the different methods to deal with them. Finally we will apply them to the astrophysical case of MWC 158 in the Section 4. 

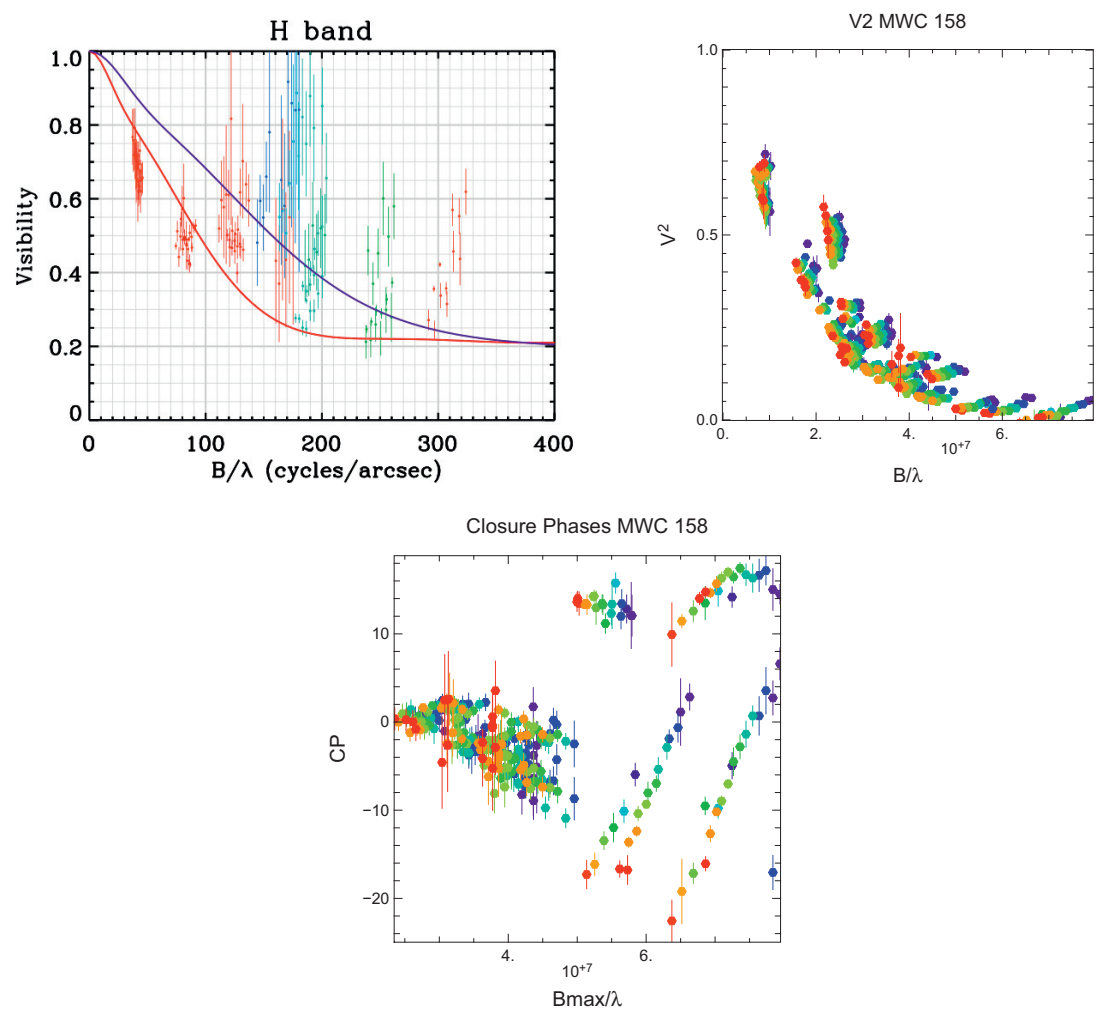

Fig. 1. Data on MWC 158. Left: AMBER (Petrov et al. 2007) data from Borges Fernandes et al. (2011). Center: PIONIER (Le Bouquin et al. 2011) squared visiblities. Right: PIONIER closure phases. For PIONIER data the gray scale varies in function of the wavelength. We can see on the visibility graphs than a "coma" trend appear for visibilities belonging to the same baseline.

\section{Chromatism}

Since interferometric instruments with spectral dispersion exist, we need to take into account the flux variations with the wavelength in order to correctly analyze the data and have access to the spectral super synthesis. In the case of Young Stellar Objects (YSOs), we noticed that the visibilities have a strong spectral dependence such as the geometrical shape of the object could not explain it.

For Herbig AeBe star, the chromatic effect explained in the Section 1 exists typically for the Near Infrared interferometry. In the following, we explore the possibility that this effect is caused by a different spectral index between the central star and its surrounding media.

In order to confirm that, we made a simple model with a central star and its dusty disk. 


\subsection{The star}

In our model, the star is considered to be unresolved. This hypothesis is justified for the young objects we are looking at. To simplify our model, we assume $V_{\text {star }}=1$.

For the star we have 3 parameters: the radius $\left(R_{*}\right)$, the distance $(d)$ and the temperature $\left(T_{*}\right)$. If we asumme a Herbig AeBe star with a temperature of $12000 \mathrm{~K}$ radiating as a black body, we know that in NIR we will look on the Rayleigh-Jeans regime of a black body (see Fig. 2). That means that the spectral curve is proportional to a power law: $F_{\lambda}^{\text {star }} \propto \lambda^{-4}$ ( $F$ being the luminous flux).

\subsection{The disk}

The disk model is simple: it is a geometrically thin optically thick passive disk. Its temperature is a function of the radius:

$$
T(r)=T\left(r_{0}\right)\left(\frac{r}{r_{0}}\right)^{-q}
$$

with:

$$
q=\frac{3}{4}
$$

see Adams et al. (1988); Lynden-Bell \& Pringle (1974).

The disk will be sampled on several rings, each ring having its own temperature as a function of its distance to the star. We will use a lot of rings (more than 100) to model the disk. We can then approximate that each ring has the fourier transform of an infinitesimal width ring. However, for the flux, we will take each ring width into account. The other geometrical parameters are the inclination (inc), the inner and outer rims radii $\left(R_{\text {in }}, R_{\text {out }}\right)$, and the temperature of the inner $\operatorname{rim}\left(T_{\text {in }}\right)$. The flux of each ring will be a black body at the temperature of the disk. The ring visibility is defined as follows (Berger 2003):

$$
V_{\text {ring }}=J_{0}\left(2 \pi r \frac{B}{\lambda}\right) \text {. }
$$

Then to obtain the Fourier transform of the whole disk we have to add the flux of each ring and sum every contributions:

$$
V_{\text {disk }}=\sum_{i}^{n_{\text {ring }}} J_{0}\left(2 \pi r_{i} \frac{B}{\lambda}\right) B_{\lambda}\left(T_{i}\right) S_{i} .
$$

$S_{i}$ being the surface of the $i$-th ring $\left(S_{i}=2 \pi r_{i} w_{i} ; w_{i}\right.$ being the width of the $i$-th ring and $r_{i}$ its radius).

In the results shown in Figure 2 the chromatic effect which tends to look like the data shown in Figure 1. The visibilities have the particularity to rise for every base even though the general trend of the visibilities is to decrease. It is similar 


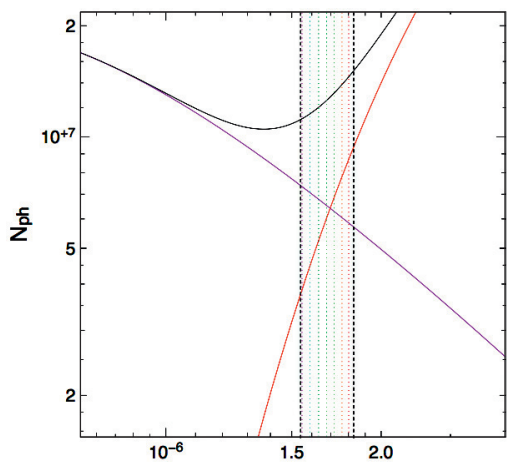

$\lambda$

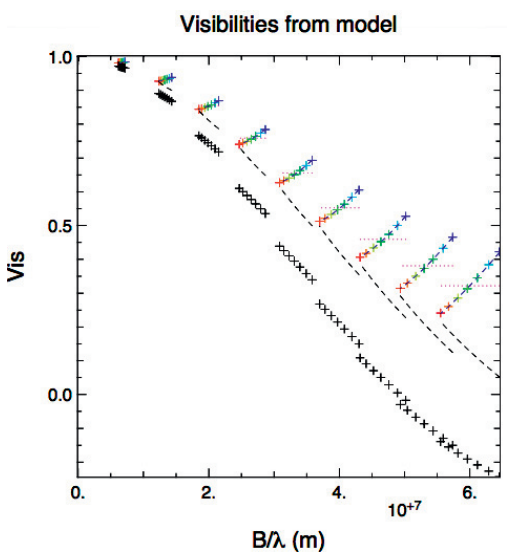

Fig. 2. On the left, we show the location of the PIONIER spectral channels on the Spectral Energy Distribution (SED) of the model. The component on the left is the stellar photosphere approximated by a black body and the component on the right is the environment. The black line is the sum of the two components. We can see that they are located at the crossing between the stellar and the dust fluxes. On the right, we can see that the chromatic phenomenon is reproduced in the visibilities. For any base the visibility is rising with the spatial frequency $(B / \lambda)$. On black we have the visibilities of the environment only.

to that we observe in real data. We can reproduce this effect by the chromatism that exists between the star and its environment. We can then suggest that the chromatic effect is not instrumental but astrophysical.

The effect is dominated by the flux ratio which is changing through the different spectral channels. If we compute the total correlated flux we have (given that the Fourier transform is linear):

$$
V_{\text {tot }}(B / \lambda) F_{\text {tot }}(\lambda)=F_{*}(\lambda)+V_{\text {disk }}(B / \lambda) F_{\text {disk }}(\lambda)
$$

with:

$$
F_{\text {tot }}(\lambda)=F_{*}(\lambda)+F_{\text {disk }}(\lambda) .
$$

If we introduce the stellar to total flux ratio $f_{*}$, we obtain the mathematical description of the chromatic phenomena:

$$
V_{\text {tot }}(B / \lambda)=f_{*}(\lambda)+V_{\text {disk }}(B / \lambda)\left(1-f_{*}(\lambda)\right)
$$

with

$$
f_{*}(\lambda)=\frac{F_{*}(\lambda)}{F_{\text {tot }}(\lambda)} .
$$

In the next section we will discuss the different methods to overcome the chromatic effect. 


\section{Methods}

Our goal is to be able to analyze chromatic data. We developed three complementary methods to do that: gray image reconstructions, data modification and parametric fit. The first two methods are based on image reconstruction and the last one is model fitting. We are mostly interested in the disk around the star and we are looking for informations on the resolved geometry and the strength of the chromatic effects.

\subsection{Image reconstruction per spectral channel}

Once we are aware of the chromatic effect, one can make image reconstructions selecting only one wavelength per reconstruction (see Fig. 3). In that case the gray image reconstruction is justified. The technique is to have one image per wavelength and to stack all the images in order to have the final broadband one.

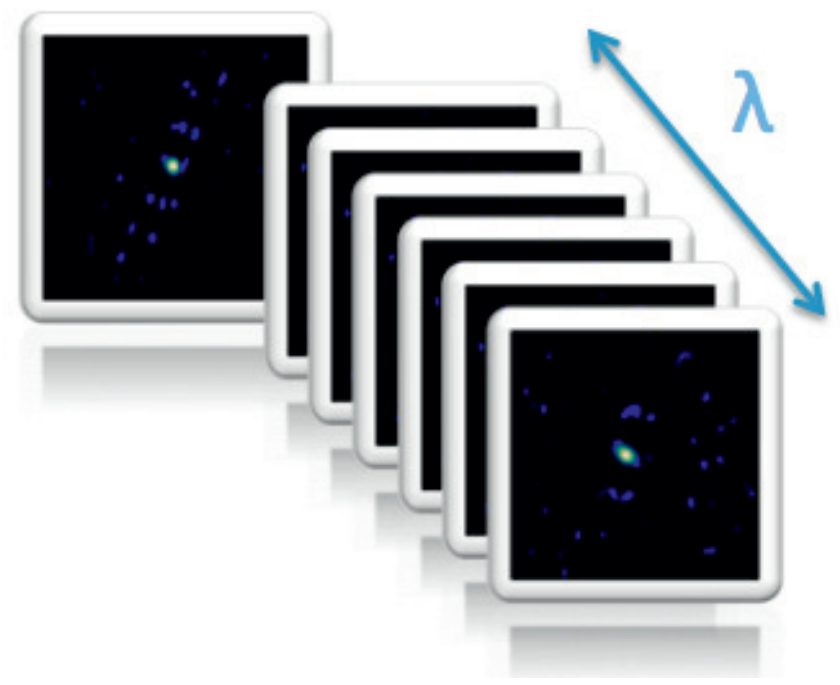

Fig. 3. An image reconstruction is made for each spectral channel of the instrument. Then all the images are stacked together in order to obtain the final image.

The presence of various components of different spectral indexes prevents from using a gray emission approximation in the image reconstruction process. As a consequence, since we need to work on a per-spectral-channel basis the $(u, v)$ coverage quality is severely affected.

\subsection{Modification of the data}

We want to have access to the disk visibilities. From the Equation (2.7), if we know the SED and then the stellar flux ratio $f_{*}(\lambda)$ and its variation through the 
wavelengths, we can compute the disk visibilities as:

$$
V_{\text {disk }}(B / \lambda)=\frac{V_{\text {tot }}(B / \lambda)-f_{*}(\lambda)}{1-f_{*}(\lambda)} .
$$

We can apply the modification described in Equation (3.1) to one of the interferometric measurements which is the power spectrum $\left(V V^{*}=|V|^{2}\right)$. In summary, our measurements are $\left|V_{\text {data }}\right|^{2}$ and we want to recover $\left|V_{\text {disk }}\right|^{2}$. Using the Equation (3.1), we have:

$$
V_{\text {disk }}^{2}(B / \lambda)=\left(\frac{\sqrt{\left|V_{\text {data }}(B / \lambda)\right|^{2}}-f_{*}(\lambda)}{1-f_{*}(\lambda)}\right)^{2} .
$$

One of the problem is the value that we take for $\sqrt{\left|V_{\text {data }}(B / \lambda)\right|^{2}}$; we must choose between the positive (phase $\phi=0)$ and the negative one $(\phi=\pi)$. But it could be solved analyzing more precisely the data and other interferometric observables like the phase of the bispectrum (also called the closure phase). The goal is to find where the visibilities are reaching the first "zero", where there is a jump of $\pi$ in the phase (and the in the closure phase). Moreover the chromatic parameter $f_{*}$ must be estimated from other observations (e.g. photometry).

It is not possible to retrieve the bispectrum phase of the dust from the data because we are loosing the phase of each pair of telescopes. The equations lead to a solution where we need the phase (Ragland et al. 2012).

\subsection{Parametric model}

In this section we have attempted to model the object. The model is geometrical and includes the chromatic effect as described in the Sections 1 and 2. Our model is composed of multiple components and was developed when chromatic data was fitted.

\subsubsection{Geometric part of the fit}

The first component of the model is an unresolved star (a dirac in the image space) which can be shifted compared to the image photo center (that will produce a rise of closure phases). The second component is a Gaussian ring. This shape is close to the shape of a puffed-up inner disk rim model. (Isella \& Natta 2005). In the Fourier space the ring is defined as in Equation (2.3) but using $\sqrt{u^{2}+v^{2}}$ for the spatial frequencies $(B / \lambda)$ and their orientations that we want to solve.

We take into account the Position Angle (P.A.), which is defined from the North to the East, and the inclination (inc). One of the parameters of this shape is the ring radius $r$. But this will define a ring with a infinitely small width. In order to have a Gaussian width we have to convolve the ring formulae by a Gaussian, in other words, to multiply the visibility of the ring by the visibility of the Gaussian function with the correspondent width $w$. Once we have the Gaussian ring, we will 
add some azimuthal modulations of the ring intensity to be closer to the physics of an inner rim. The modulations are functions in cosinus and sinus of the azimuthal angle $(\alpha)$ of the ring which starts at its major axis. We have included two sorts of modulation: one on $2 \pi\left(c_{1}, s_{1}\right)$ and the second on $\pi\left(c_{2}, s_{2}\right)$. They are described on Figure 4 We add a Gaussian width to the ring, with $r_{\text {gauss }}$ being the Half Width at Half Maximum (HWHM) of the Gaussian function.
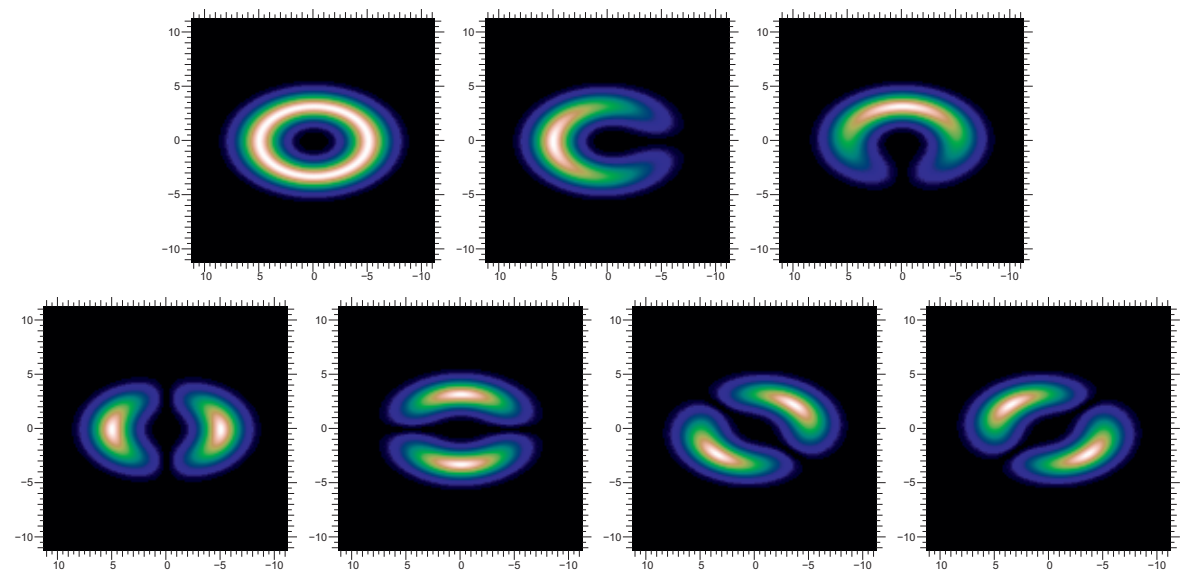

Fig. 4. Description of the azimuthal modulations. The first figure is the original gaussian ring slightly inclined. The second figure represent a $\pi$-modulation with $c 1=1$ (modulation through the long axis) and the third with $s 1=1$ (modulation through the short axis). The second line represent the $2 \pi$-modulation with respectively $c 2=1, c 2=-1$, $s 2=1, s 2=-1$. The model can combine the modulations and express them with slighter intensity (below 1).

We have a model with three components: the star, the Gaussian ring and a second Gaussian ring or a Gaussian function.

The total visibilities are depending on all these components weighted by their flux.

\subsubsection{Modeling the chromatism}

To obtain the model visibilities we use the linearity property of the Fourier transform.

$$
F_{\text {tot }} V_{\text {tot }}=F_{*} V_{*}+F_{1} V_{1}+F_{2} V_{2} .
$$

The fluxes are the ones recieved by the interferometric instrument. So we have:

$$
F=\int_{\lambda-\frac{\Delta \lambda}{2}}^{\lambda+\frac{\Delta \lambda}{2}} F_{\lambda} d \lambda=\int_{\nu-\frac{\Delta \nu}{2}}^{\nu+\frac{\Delta \nu}{2}} F_{\nu} d \nu
$$

with $\Delta \lambda$ and $\Delta \nu$ being the spectral width of one spectral channel in wavelength $(\lambda)$ and frequency $(\nu)$. 
We will use the approximation that the channel spectral width is constant and that the flux is constant in one spectral band. The flux is then equal to the value of $F_{\lambda}$ at the central wavelength of a spectral channel. From the Equation (3.3), we see that we can determine a flux ratio at one wavelength and to deduce the ratios on the other wavelength by the laws that we assume for each component. PIONIER is operating in the NIR in the $\mathrm{H}$ band. At this wavelength, we can assume that Herbig stars are in their Rayleigh-Jeans regime. That means that their flux $F_{\lambda}$ is proportional to the wavelength at the power of -4 . The laws for the environment are more difficult to find. We can fit a power-law in wavelength or to a black body variation if we are resolving a thermal emitting region. Since the dust temperature is supposed to be below 2000 K (Dullemond \& Monnier 2010), we can assume that it is in its Wien regime. Then if we assume black body regimes we obtain (from Eq. (3.3)):

$$
V_{\mathrm{tot}}(B, \lambda)=f_{*}^{0}\left(\frac{\lambda}{\lambda_{0}}\right)^{-4}+f_{1}^{0} \frac{B B\left(\lambda, T_{1}\right)}{B B\left(\lambda_{0}, T_{1}\right)} V_{1}(B B, \lambda)+f_{2}^{0} \frac{B B\left(\lambda, T_{2}\right)}{B B\left(\lambda_{0}, T_{2}\right)} V_{2}(B B, \lambda)
$$

with $f^{0}$ the flux ratios at $\lambda_{0}, T$ the temperature of a component and $B$ the baseline and

$$
B B(\lambda, T)=\frac{2 h c \lambda^{-5}}{\exp \frac{h c}{k_{B} \lambda T}-1}
$$

is the black body function, with $h$ the Planck constant, $c$ the light speed, and $k_{B}$ the Boltzmann constant.

The variations of the flux ratios through the observational band will build the chromatic effect that we want to take into account in our fit.

Once we get all our tools to investigate data with chromatic effect, let us apply them on an astrophysical case: MWC 158.

\section{The case of MWC 158}

The interest on this object came with the data we get with PIONIER (Le Bouquin et al. 2011) a 4 telescopes interferometric, visitor instrument operating at the VLTI and which observes in the $\mathrm{H}$ band.

\subsection{Image reconstructions}

We were interested into this data (see Fig. 1) because it shows clearly signs of chromatism. As the $u, v$-plan is sufficiently covered we can reconstruct images. We use the Mira algorithm (Thiébaut 2008), but as many image reconstruction algorithms it does not take into account the chromatism. Since it extrapolates and interpolates the Fourier space, the chromatism makes him "guessing" badly and many artifacts appear. We then use the monochromatic reconstructions per spectral channel. It means that we select every spectral channel one by one and 

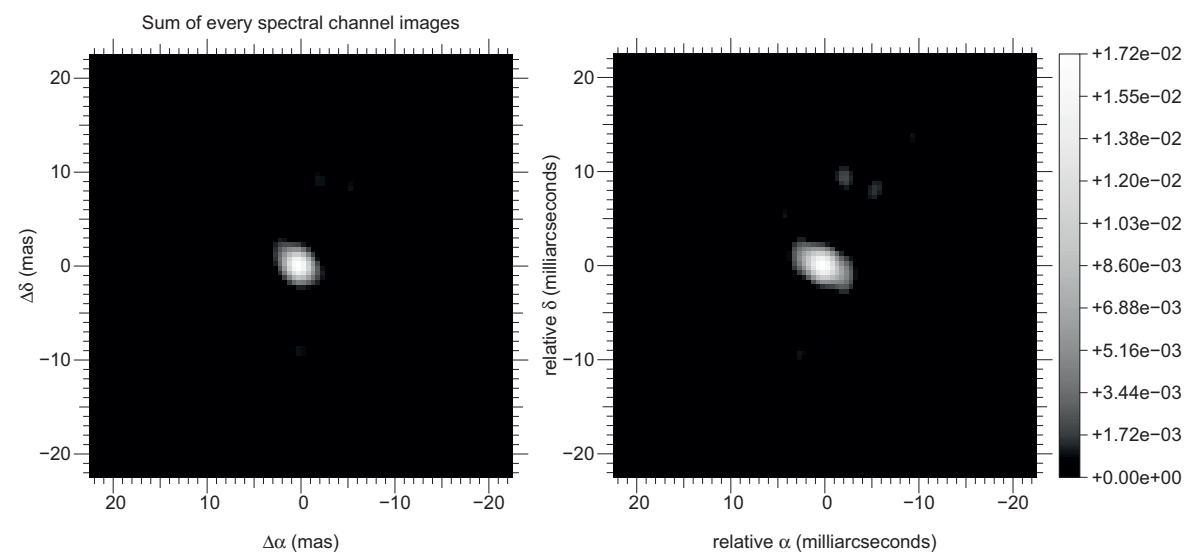

Fig. 5. Left: the stack of image reconstructions per spectral channels. Right: image reconstruction after modifying the squared visibilities.

make one reconstruction by channel. We also use the visibility correction method. These method (described Sect. 3.2) is not modifying the closure phases (they should be stronger). The results are showed on the Figure 5 .

We can see that there is a second resolved component. We can also see the orientation of the smallest extended component. Both of the reconstruction methods shows similar patterns. That brings us to the idea to fit two extended components.

\subsection{Parametric fit}

The fit bring us an idea on the geometry and the light emission from the source but with a strong a priori which is the model we want to fit. That is why we took the geometries suggested by the image reconstructions. We can see a central extended part which is composed of the star and its environment which seems to have a P.A. and an inclination.

We have begun the fit with one extended component which is a Gaussian function or a Gaussian ring. Both of the fits gave us the more or less the same inclinations and P.A. which are consistent with the image reconstructions. But the data was not entirely fitted: the short baselines indicates that there is a more extended component as showed by image reconstruction. We then add another component to our fit. In order to fit the strong closure phase signal we add azimuthal modulation to the ring. It appears not to be sufficient, and the best fit was to shift the central star. It is the only solution to fit the closure phases.

In the end, and adding the different parameters, we ended with 15 parameters and a $\chi^{2}$ of 3.5. In the current state of the data processing and interpretation, we believe that the best fit is presented Figure 6 . The parameters are in the Table 1. We can see that the best fit is done with two Gaussian rings. 


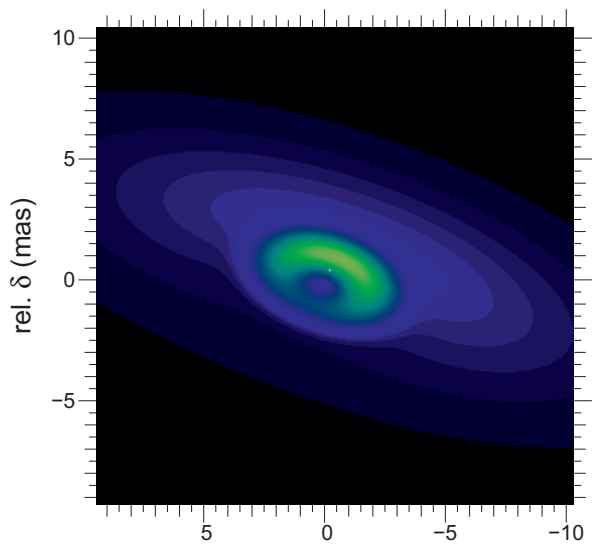

rel. $\alpha$ (mas)

V2 fit

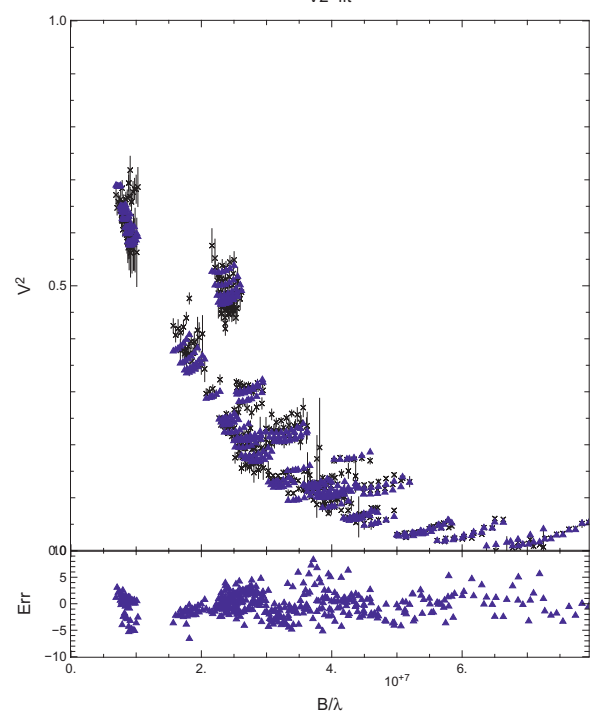

Closure phase

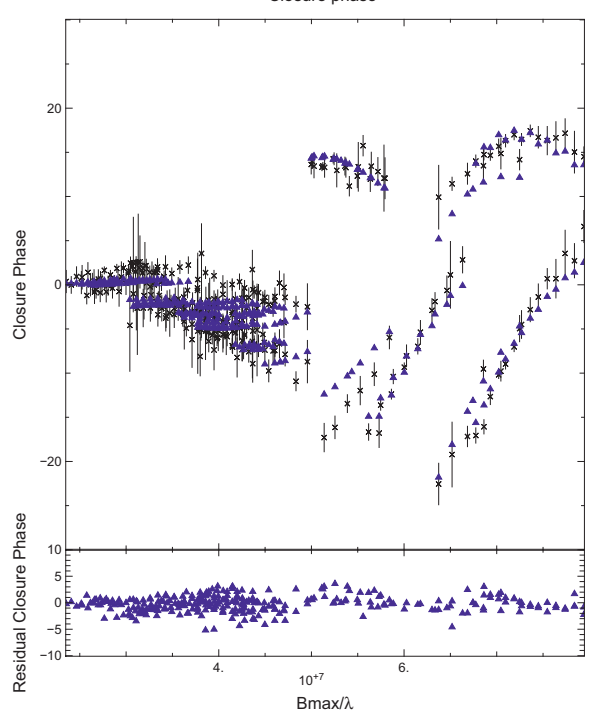

Fig. 6. The best fit results are presented. Left: the image corresponding to the best fit. Center: the fit on the V2. Left the fit on the Closure Phases. The triangles are the fit result, the crosses are the data points.

The geometrical fit suggests a star, with a relatively close Gaussian ring (radius of 1.5 mas) with a lot of flux $(\approx 60 \%)$. We interpret that as the resolution of the inner rim of the dusty disk. Its azimuthal modulation is strong in the semi minor axis direction which leads us to deduce that it is due to the inclination. Moreover, the star is shifted towards the most brilliant part of the inner rim. It indicates that the inner rim has a non-negligible height. The outer ring suggests the continuation of the disk, or a part of the disk which is not self shadowed, or a halo. The constraints are poor so we can not conclude on its origins. 


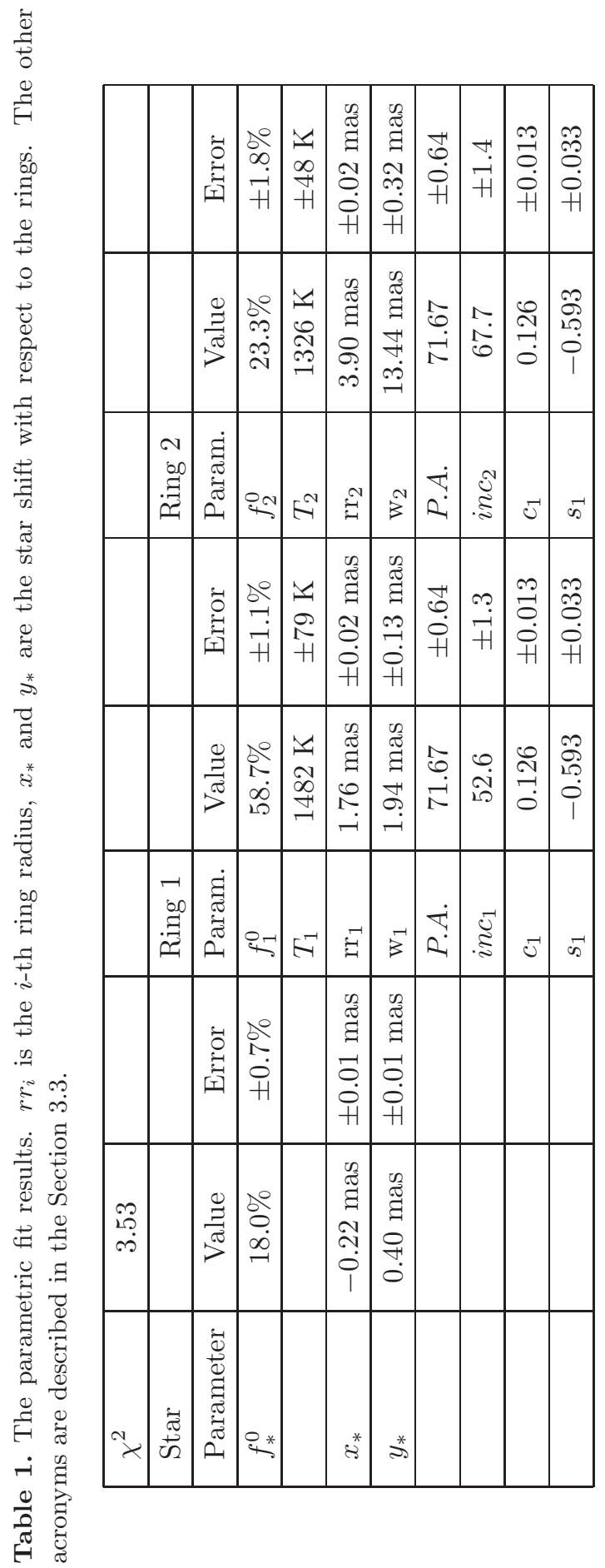


Table 2. The previous interferometry results on MWC 158. Some results were complete on instruments watching at longer wavelengths $(10.7$ or $2.2 \mu \mathrm{m})$. The P.A. are consistent and the inclinations $i$ also. The references are: 6: Borges Fernandes et al. (2011) and 20: Monnier et al. (2009).

\begin{tabular}{|c|c|c|c|c|c|l|}
\hline FWHM & FWHM2 & $i$ & $P . A$. & $\chi^{2}$ & $\lambda_{0}$ & Ref. \\
\hline $66 \pm 4$ & & 45 & $63 \pm 6$ & 1 & $10.7 \mu \mathrm{m}$ & 20 \\
\hline $64.7 \pm 0.6$ & & $70.1 \pm 0.7$ & $59.1 \pm 1.7$ & 5.1 & $10.7 \mu \mathrm{m}$ & 6 \\
\hline $35.2 \pm 1.5$ & $131.4 \pm 11.2$ & $56.7 \pm 0.4$ & $65.9 \pm 2.0$ & 1.9 & $10.7 \mu \mathrm{m}$ & 6 \\
\hline $4.4 \pm 0.5$ & & $54 \pm 8$ & $66 \pm 9$ & 40.8 & $2.2 \mu \mathrm{m}$ & 6 \\
\hline $3.0 \pm 0.4$ & $\geq 14.0$ & $54 \pm 8$ & $77 \pm 2$ & 13.3 & $2.2 \mu \mathrm{m}$ & 6 \\
\hline
\end{tabular}

The results are shown in Table 2. They are closed to the images get by reconstruction. The results are also consistent with that found with previous observations (Borges Fernandes et al. 2011; Monnier et al. 2009). The authors found similar P.A. with close values of the inclination of the most luminous extended object.

The fit of the chromatism, indicates us a black body temperature of the inner $\operatorname{rim}$ of $\approx 1500 \mathrm{~K}$ (see Table 1 ). This is approximately the dust sublimation temperature found in the litterature (Dullemond \& Monnier 2010; Duschl et al. 1996).

\section{Conclusions}

The chromatic effect due to the flux predominances of two objects of different sizes is well understood and can be used in order to find astrophysical information of the object. In the case of Herbig AeBe stars we are able to have an approximation of the temperature of the components. If the chromatic information is given, we can perform gray disk image reconstructions. They are contributing to the astrophysical analysis of the object because they show the P.A. and the inclinations of the disk. Moreover, in the case of MWC 158 it brought us the idea of the second extended component, even if it is poorly constrain (we can have the information on the flux ratio). By the fit we were able to find a value for the inner rim radius and its temperature and to compare what we found with the data from photometry. We bring the first confirmation of the dust sublimation temperature at the inner rim. The information taken from the NIR interferometry and the chromatic effect argue in favor of a young nature of MWC 158.

The main challenge is to be able to make chromatic Young Stellar Objects image reconstructions keeping the super spectral synthesis and without information on the total flux variation. It seems to be degenerated and we need information from photometry. One of the thing which is in process of testing, is the adaptation of the Mira algorithm to the case of young stellar object. The "gray" mira free parameters are the image pixels intensities. If we define the image as the 
image of the dust at $\lambda_{0}$, the start can be represented by a dirac at the center of the image. Hence, we can put the stellar flux and a stellar relative spectral power law as additional parameters to the fit. Since the regularization will tend to smooth the Fourier plan, the algorithm will favor the added parameters to fit the fixture. Indeed, the parameters will not be constrained by the regularization and the gradient will be stronger on it than on the pixels values.

For these objects, there is a need to include parametric models in the image reconstruction algorithms and to develop a global chromatic image reconstruction algorithm.

This work is supported by the French ANR POLCA project (Processing of pOLychromatic interferometriC data for Astrophysics, ANR-10-BLAN-0511).

\section{References}

Adams, F.C., Lada, C.J., \& Shu, F.H., 1988, ApJ, 326, 865

Andrillat, Y., \& Houziaux, L., 1991, IAU Circ., 5164, 3

Benisty, M., Natta, A., Isella, A., et al., 2010, A\&A, 511, A74

Berger, J.-P., 2003, EAS Publications Series, 6, 23

Borges Fernandes, M., Kraus, M., Chesneau, O., et al., 2009, A\&A, 508, 309

Borges Fernandes, M., Meilland, A., Bendjoya, P., et al., 2011, A\&A, 528, A20

Cabrit, S., 2003, Ap\&SS, 287, 259

Cabrit, S., Ferreira, J., Dougados, C., \& Garcia, P., 2010, Highlights of Astronomy, 15, 261

Dougados, C., Bouvier, J., Ferreira, J., \& Cabrit, S., 2005, IAU Symp., 226, 491

Dougados, C., Cabrit, S., Ferreira, J., et al., 2004, Ap\&SS, 293, 45

Dullemond, C.P., \& Monnier, J.D., 2010, ARA\&A, 48, 205

Duschl, W.J., Gail, H.-P., \& Tscharnuter, W.M., 1996, A\&A, 312, 624

Eisner, J.A., Graham, J.R., Akeson, R.L., \& Najita, J., 2009, ApJ, 692, 309

Hutsemekers, D., 1985, A\&AS, 60, 373

Isella, A., \& Natta, A., 2005, A\&A, 438, 899

Le Bouquin, J.-B., Berger, J.-P., Lazareff, B., et al., 2011, A\&A, 535, A67

Lynden-Bell, D., \& Pringle, J.E., 1974, MNRAS, 168, 603

Malbet, F., Benisty, M., de Wit, W.-J., et al., 2007, A\&A, 464, 43

Monnier, J.D., \& Millan-Gabet, R., 2002, ApJ, 579, 694

Monnier, J.D., Tuthill, P.G., Ireland, M., et al., 2009, ApJ, 700, 491

Petrov, R.G., Malbet, F., Weigelt, G., et al., 2007, A\&A, 464, 1

Pogodin, M.A., 1997, A\&A, 317, 185

Ragland, S., Ohnaka, K., Hillenbrand, L., et al., 2012, ApJ, 746, 126

Tannirkulam, A., Monnier, J.D., Harries, T.J., et al., 2008, ApJ, 689, 513

Tatulli, E., Isella, A., Natta, A., et al., 2007, A\&A, 464, 55

Thiébaut, E. 2008, in Society of Photo-Optical Instrumentation Engineers (SPIE) Conf. Ser., Vol. 7013

van Leeuwen, F., 2007, A\&A, 474, 653 